\title{
Electromagnetic brakes in electromechanical actuators for aerospace applications
}

\author{
Moreno D'Andrea \\ $R \& D$ Department \\ UMBRAGROUP spa \\ Albanella, Italy \\ mdandrea@umbragroup.com \\ Davide Macera \\ $R \& D$ Department \\ UMBRAGROUP spa \\ Albanella, Italy \\ dmacera@umbragroup.com
}

\author{
Giovanni Di Domenico \\ $R \& D$ Department \\ UMBRAGROUP spa \\ Albanella, Italy \\ gdidomenico@umbragroup.com \\ Francesco Fiume \\ $R \& D$ Department \\ EMS srl \\ Albanella, Italy \\ ffiume@electroms.it
}

\begin{abstract}
More Electric Aircraft has been a well-known term for a few years now. Key components for the More Electric Aircraft are the electromechanical actuators, these most of time among the other components as electric motors, sensors, mechanical components, etc. have also electromechanical brakes. This paper describes the design process of an electromechanical brake for an aerospace application and the experimental tests done to verify congruency among real electromagnetic forces and those evaluated by Finite Element Analysis.
\end{abstract}

Keywords-More Electric Aircraft, Brake, Electromechanical Brake, Electromagnetic brake, Aerospace applications.

\section{INTRODUCTION}

The needs of improvements in aircraft performances and reliability, reduction of fuel consumption, gas emissions and of operating and maintenance costs drove to the concept of More Electric Aircraft (MEA) and recently All Electric Aircraft (AEA). The first objective behind the concepts of MEA and AEA is substitution of traditional actuators, pneumatic and hydraulic, with Electro-Mechanical Actuators (EMAs).

EMAs are used/will be used in several applications on aircrafts such as primary and secondary flight surfaces control [1],[2],[3], landing gears and on secondary systems as opening doors and food trolley elevators and others.

The more complex EMA is an electromechanical device equipped with electric motor, sensors, integrated Electronic Control Unit (ECU), electromagnetic brake and other mechanical components.

Electromechanical brakes are commonly used in several aerospace and industrial applications [4], of course in the former applications electromagnetic brake consist on a potential component adopted in electromechanical actuators and so they must contribute to the achievement of the objectives at the basis of EMAs for MEAs, that is significant improvements in terms of weight and volume reduction, total life cycle costs, maintainability and aircraft reliability. A further important requirement that an electromagnetic brake of an EMA must have is the complete absence of interferences with sensors.

This paper focus on electromagnetic brakes for electromechanical actuators in aerospace applications. Section II gives an overview of different electromechanical brake's

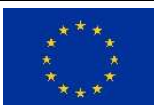

Clean Sky The project leading to these results has received funding from the Clean Sky 2 Joint Undertaking under the European Union's Horizon 2020 research and innovation programme under Grant Agreement no. 945548 - GAM-2020-REG (Amdt. Ref. No AMD945548-3) typologies. Section III discusses the design process of electromagnetic brakes, Section IV gives more details on the electromechanical brake for Winglet/Wingtip (WL/WT) applications. Section V discusses the test on the brake's prototype. In conclusion a comparison of electromagnetic forces among those evaluated by finite element analysis (FEA) and experimentally verified on the prototype is done.

\section{ELECTROMECHANICAL BRAKES OVERVIEW}

Several aerospace applications, especially those for flight control applications, use electromechanical actuators equipped with electromagnetic brakes. The brake's functionality can be different: stationary, functional or safety. Different brake topologies are available, these are shown in Fig. 1, Fig. 2, Fig. 7.

These brakes can be distinguished in two categories: static brakes and dynamic brakes. The former category embraces solenoid pull brakes and toothed brakes, the latter embraces clutch disk brakes.

Static brakes engage when the actuators are already stopped so with no motion, dynamic brakes can engage also with the actuators in motion and allow to reduce speed of the actuators dynamically. In terms of braking torque static brakes ensure a greater one than the dynamic ones within the same space envelope.

In addition to the difference in topology, electromagnetic brakes can be of the power-on or power-off type. On and Off refer to the status of the power supply in the condition which the brake is normally engaged so a power-off type brake is normally engaged (system braked) while is disengaged under supply voltage, vice versa a power-on type brake is normally disengaged (system free to move) while is engaged under supply voltage.

About the supply, generally electromechanical brakes are supplied with two voltage levels, the greatest insists on the release phase, the lowest insists in the maintain phase. 


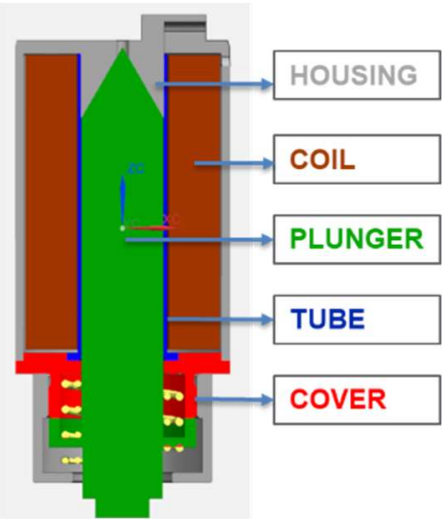

Fig. 1. Example of solenoid pull brake.

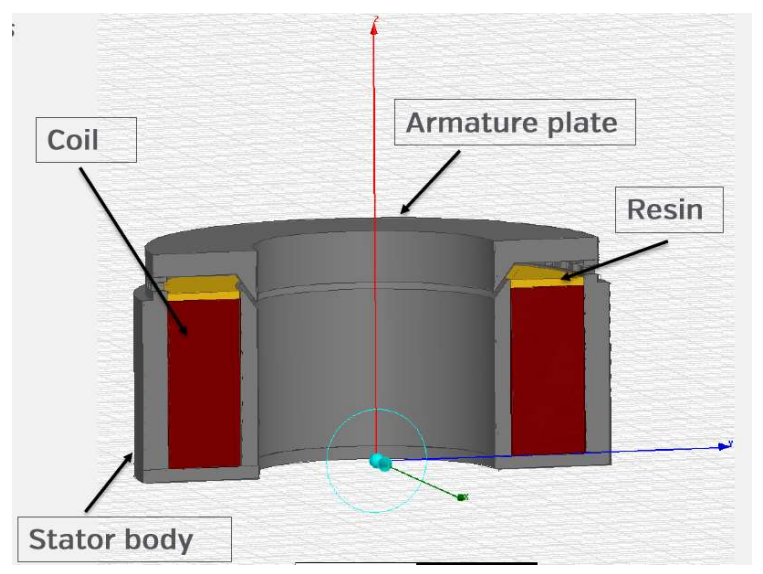

Fig. 2. Example of toothed brake.

\section{EleCtromechanicAl BRAKE's DESIGN ProcesS}

The design of an electromechanical brake is a design for steps that can be identified in three main steps: mechanical, electromagnetic and thermal respectively. Generally, an iterative process is required to find the right dimensioning compromise. Regardless the brake's topology the goals are to ensure that the electromagnetic force delivered by the brake is greater than the force exerted by the springs and that the braking torque generated by the clutch disks block the system in a determinate position.

Mechanical and thermal aspects of the brakes' design process are not treated in this paper because it focuses on the electromagnetic aspects of electromechanical brakes. Shortly, mechanical study has the goal to choose the brake suitable for the application in terms of topology, springs and envelope based on force density and dynamic requirements. Mechanical study requires several analytical and FEA verifications. Thermal study is essentially a verification performed by FEA software when the electromagnetic study is almost completely defined to ensure that the maximum permissible temperatures, i.e. insulation class, of brake's components are respected in all operating conditions considering the losses of the brake itself.

Electromagnetic design process of electromechanical brakes can be divided in different phases summarized in Fig. 3.

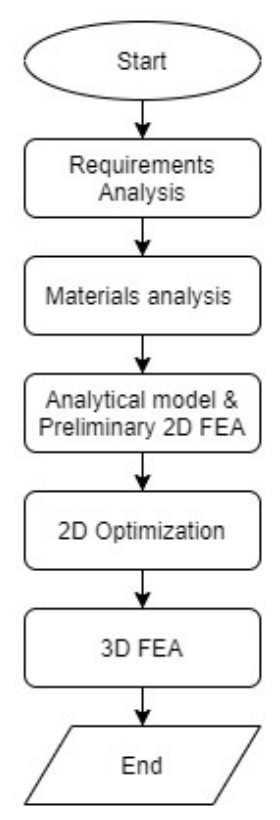

Fig. 3. Electromagnetic design process of electromechanical brakes.

The first phase consists on the requirement analysis, among requirements the most important for the electromagnetic brake's design are operating temperature range and electrical constraints in terms of current, voltage, power.

An important aspect to consider for the electromagnetic brake design is the material choice: magnetic properties such as relative permeability and saturation flux density are very important but also mechanical properties must be taken in account especially for some specific brake's topologies i.e. tangential tensile strength in toothed brakes. Generally, core and anchor in electromagnetic brakes for aerospace applications are made in cobalt soft magnetic alloy because of its high magnetic saturation. Apart from the magnetic properties of core and anchor materials also the materials of the other components of the electromechanical brakes and the surrounding components in the actuator are important and they must be considered in the design phase and in FEA evaluation because they could affect the electromagnetic forces developed and the other performances.

Subsequently to the above steps, an analytical model of the brake is developed on the basis of the preliminary geometry of the brake itself to identify the potential characteristics of the coil in terms of number of Ampere turns, i.e., number of turns and AWG of the elementary turn and so of electromagnetic force at different airgaps. About airgaps, generally the first design phases limit to consider the worst case in terms of electromagnetic force and so the condition at maximum airgap corresponding to the maximum reluctance condition. About analytical models, most of the time they don't consider the magnetic saturation and they limit to consider the common components between the different brake's topologies, i.e., core, coil, anchor, so a preliminary FEA analysis is unavoidable.

2D FEAs allow to give more accurate results than those deriving from the analytical model and allow to consider the other ferromagnetic materials of the brake and its surroundings. With a FEA model, basing on the flux lines distribution, the designer can also evaluate which components 
have or not to be considered in the next design phases and to define the limit of the domain to analyze in next step.

2D model for FEA is generally a parametric model Fig. 4 where geometric dimensions of core, anchor and coil and number of turns are parametrized. In this way the model can be used for an optimization process Fig. 5. The optimization can be mono or multi objective, generally the objective is to maximize the electromagnetic force developed by the brake minimizing current density, power consumption, weights and magnetic induction, observing envelope and other constraints.

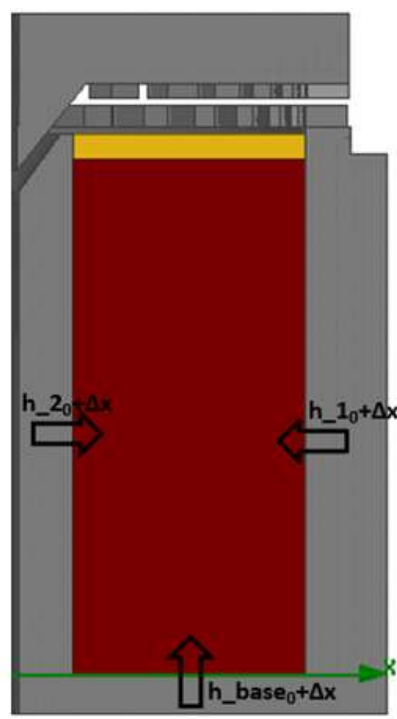

Fig. 4. Example of electromagnetic brake's parametric model.

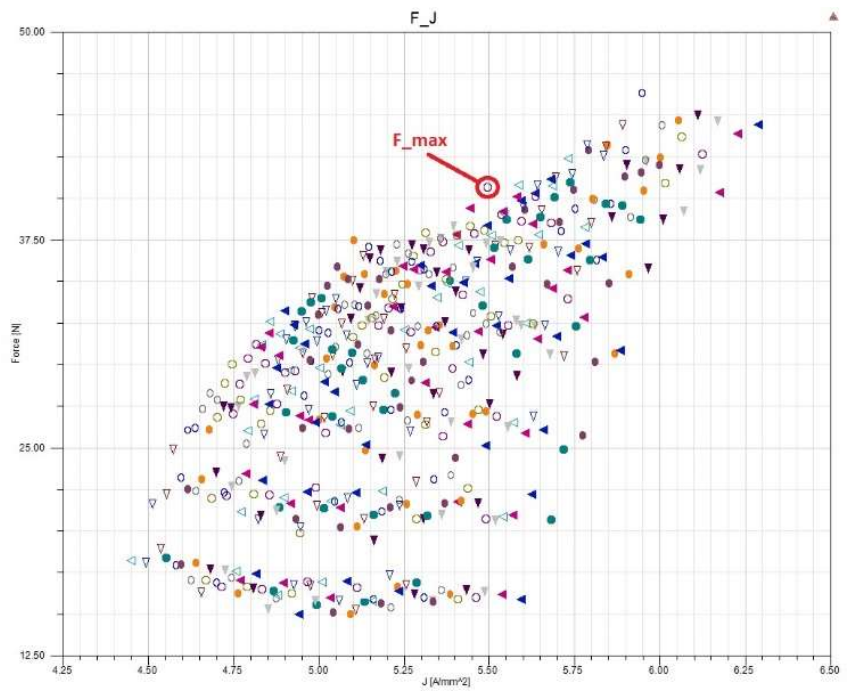

Fig. 5. Example of representation resulting from an optimization problem.

At the basis of 2D FEA model there are some simplifying hypothesis e.g. to assume that brake's geometry is axial symmetric, that's not the reality because of some dissymetries e.g. cable exit, slot casing for springs and any centring. 2D model represent a good compromise between accuracy and computational burden. About computation burden, of course the $3 \mathrm{D}$ optimization can be performed but it requires a high one.

To evaluate more accurately brake's performances and to take in account the real architecture, 3D analysis on the brake designed at the end of optimization are also performed both with only electromagnetic brake's components both with other surrounding components of the EMA.

At the end of these steps the final brake's architecture is achieved and it's possible to proceed for thermal verification analysis and then with prototyping phase and so on.

Both in 2D and in 3D several studies are performed, shortly in magnetostatic environment static performances such as electromagnetic brake's forces are evaluated in different voltage and airgap conditions. In magnetostatic environment also flux distribution and induction are evaluated Fig. 6. Transient analysis are performed to evaluate dynamic of electromagnetic brakes and remanent force, the latter can represent an insidious problem [5].

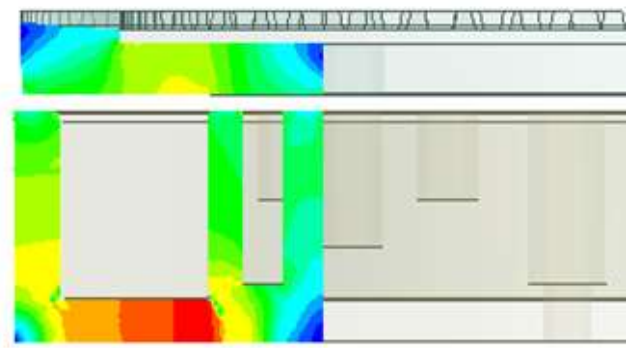

Fig. 6. Example of induction map.

\section{WingLET/WNGTIP ELECTROMECHANICAL BRAKE DESIGN}

The operation mode of an EMA for aircraft's movable surfaces, so for WT and WL too, require that the actuator shall stop the surface in a definite position and at the same time to dynamically brake the surface according a definite command position.

According to the requirements for Winglet applications, i.e. good braking capacities and high dynamics, a power-off clutch disk brake has been adopted. In fact, while on one hand the required braking torque is not excessively high, on the other it was necessary to have high dynamic properties. Moreover, since the surface is moved only on flight climb phase (landing gear up) with exclusion of Take Off, Approach and Landing phase, the brake should be braked during most of the flight. For this reason, a power-off solution was preferred [6].

Fig. 7 shows an example of clutch disk brake with components' description. 


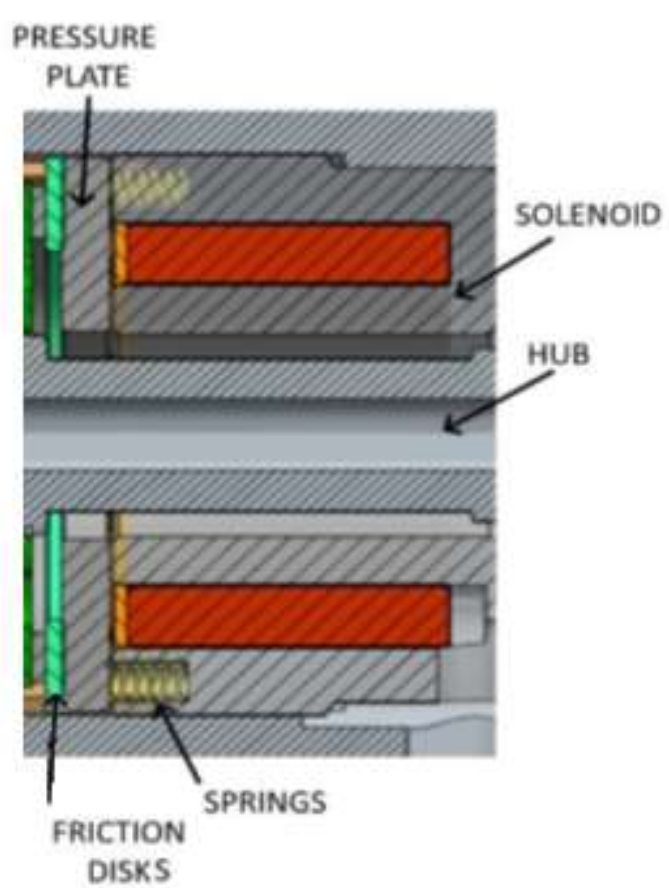

Fig. 7. Example of clutch disk brake.

Brake's operation is relatively simple: when the coil is not energized the springs press the press platr (anchor) against the friction disks thus generating a braking torque. When the coil is energized, the coil and the housing (core) generate a magnetic field and a magnetic force attract the anchor, when the magnetic force is greater than the elastic force of the springs the anchor starts to move and the airgap decreases progressively, in this way the anchor detaches from the friction disks thus allowing the actuator to move. The airgap represents the distance between core and anchor and is set according the needed preload force for the application.

For the WL/WT applications the design process described in chapter III was adopted.

For confidentiality reasons design details are not reported in this paper.

\section{ELECTROMECHANICAL \\ BRAKE PROTOTYPE EXPERIMENTAL VALIDATION}

The goal of this paper is to validate the FEA model of the electromagnetic brake. To reach this goal a comparison among electromagnetic forces resulting from FEM (Finite Elements Method) analysis and those evaluated experimentally was performed.

The test equipment is relatively simple and consist on a laboratory DC power supply, a feeler and some 3D printed tools and of course an electromagnetic brake prototype, Fig. 8 , the latter consists just in a sub-assembly composed by anchor, springs and core assembly, i.e., core, coil, insulation, wire and resin.

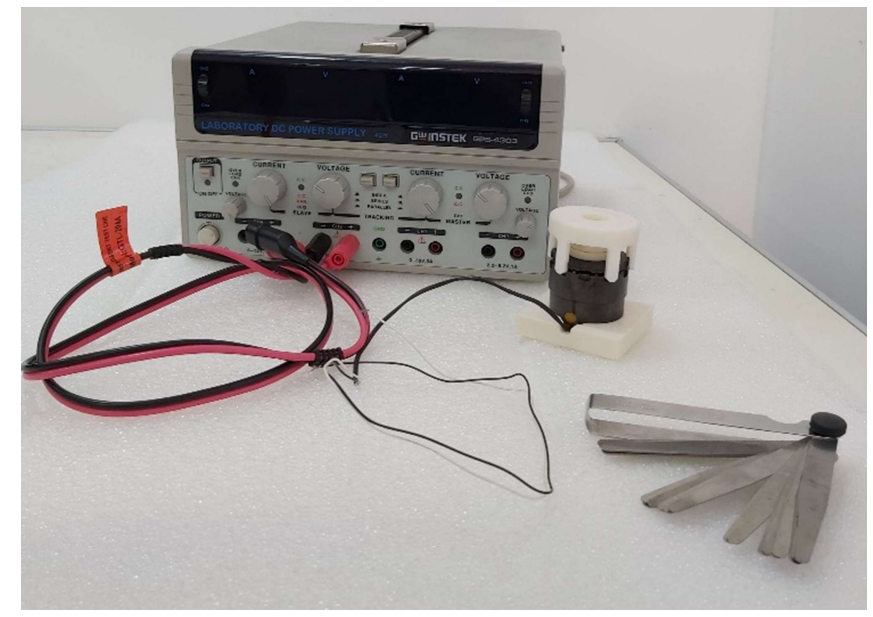

Fig. 8. Test equipment.

DC power supply was set to supply the coil with voltage and current at room temperature according to the real design, the airgap was set with the adjust nut 3D printed and verified with the feeler, alignment among anchor and core is verified by a 3D printed tool obtained modifying the brake frame 3D model Fig. 9 . To make the test sensible the airgap was set in such a way that at a lower one the brake certainly attracts the anchor and does not attract it at a greater one.

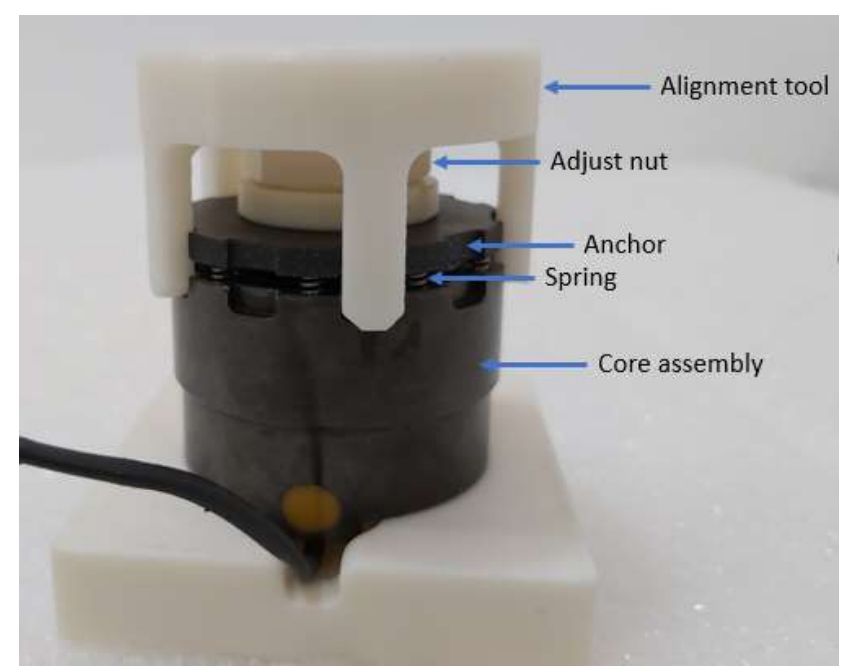

Fig. 9. Detail of test equipment: electromechanical brake and 3D printed tools.

Test conditions differ in the number of springs: the electromagnetic brake under test has ten springs equispaced radially, among a tested condition and the other two diametrically opposed springs were removed. Removing springs of course the total elastic force decreases so to restore the previous described condition the airgap was increased.

With this approach each test condition was identified with an airgap value and a number of springs, that means a quantifiable value of elastic force:

$$
F=n \cdot k \cdot\left(l_{0}-l_{\text {slot }}-g\right)
$$

Where:

$F$ is elastic force [N]

$n$ is the number of springs

$k$ is the spring stiffness [N/mm] 


\section{$l_{0}$ is the free length of the spring [mm] \\ $l_{\text {slot }}$ is the length of the spring's slot [mm] \\ $g$ is the airgap $[\mathrm{mm}]$}

To make a comparison the electromagnetic forces, magnetostatic analysis in the same supply and airgap conditions were performed, both are showed in Fig. 10.

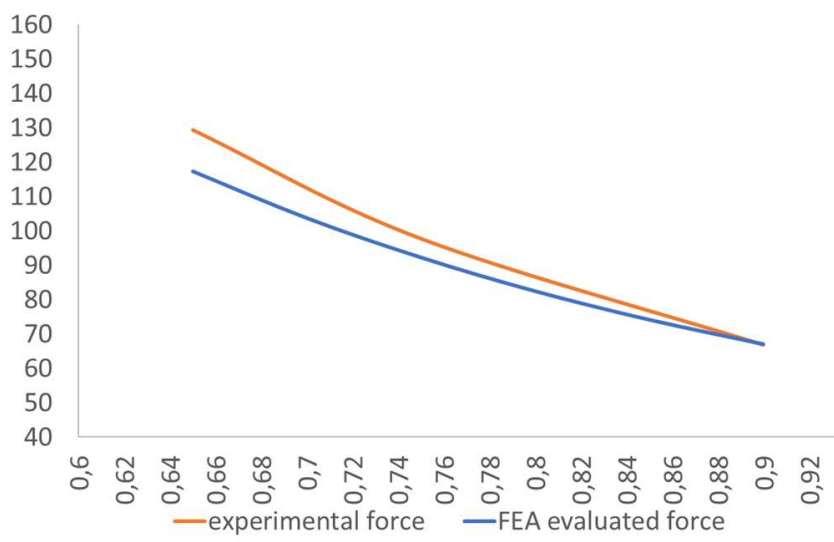

Fig. 10. Force vs airgap: experimental and FEA evaluated comparison.

Elastic forces of the spring, i.e. in this case electromagnetic brake forces, are analytically evaluated also taking in account the tolerance chain to demonstrate that brake performances can change in fact moving away from nominal conditions in terms of spring stiffness, springs' slot dimensions and springs' dimensions, the real scenarios are different from the one evaluated in nominal conditions. In Fig. 11 nominal, maximum and minimum electromagnetic brake forces are showed.

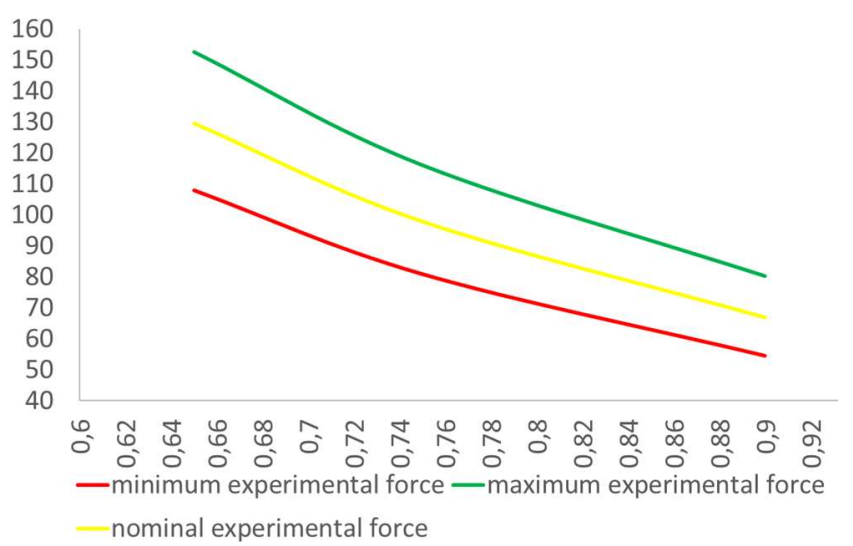

Fig. 11. Force vs airgap: experimental and FEA evaluated comparison.

\section{CONCLUSION}

The paper discusses the design process of electromechanical brakes, the topologies, fields of application. Experimental tests gave results comparable with those evaluated by FEA so the FEA model and the design is validated.

\section{REFERENCES}

[1] M. Villani, M. Tursini, G. Fabri and L. Castellini, "High Reliability Permanent Magnet Brushless Motor Drive for Aircraft Application," in IEEE Transactions on Industrial Electronics, vol. 59, no. 5, pp. 2073 2081, May 2012.
[2] M.Villani, M.Tursini, G.Fabri, L.Di Leonardo, "A switched-reluctance motor for aerospace application", ICEM 2014, XXI International Conference on Electrical Machines, p. 2073-2079, IEEE, ISBN: 9781-4799-4775-1, Berlin, September 2-5, 2014.

[3] M.Tursini, M,Villani, G.Fabri, L.Di Leonardo, "A switched-reluctance motor for aerospace application: design and analysis and results", Electric Power System Research 142, pp.74-83, Elsevier, 2017.

[4] Y. Yasa, E. Sincar, B. T. Ertugrul and E. Mese, "Design considerations of electromagnetic brakes for servo applications," 2014 IEEE 23rd International Symposium on Industrial Electronics (ISIE), Istanbul, 2014, pp. 768-774, doi: 10.1109/ISIE.2014.6864709.

[5] Jandura, L., "Brake Failure from Residual Magnetism in the Mars Exploration Rover Lander Petal Actuator", proc. 37 Aerospace Mechanisms Symposium, pp 221-235, NASA Johnson Space Center, 19-21 May 2004

[6] Dimino, I.; Gallorini, F.; Palmieri, M.; Pispola, G. Electromechanical Actuation for Morphing Winglets. Actuators 2019, 8, 42. 